\title{
Optimal embedded system for two-axis tracking PV panels
}

\section{Florin Dragomir ${ }^{1 *}$, Otilia Elena Dragomir ${ }^{2}$, Mihaela Eugenia Ivan ${ }^{3}$, Sergiu Stelian Iliescu ${ }^{4}$, Ioana Stănescu 5}

\author{
1, 2, 3 Valahia University of Targoviste, Targoviste, Romania \\ ${ }^{4}$ Polytechnic University of Bucharest, Bucharest, Romania \\ ${ }^{5}$ Advanced Technology Systems, Targoviste, Romania
}

\author{
Index Terms \\ Renewable Sources \\ PV Systems \\ PV Tracker
}

Received: 17 September 2016

Accepted: 10 October 2016

Published: 12 February 2017

\begin{abstract}
In this paper, we design and implement a two-axis tracking system of PV systems that follows the Maximum Power Point (MPP) using a programmable circuit XILINX type Complex Programmable Logic Device- CPLD and Xilinx ISE software. Thus, the PV module will reach its MPP in relation to the date and time of the day. The test bed relies on an algorithm integrated in the XILINX that has as inputs: date, location's latitude and longitude, the standard longitude (related to the location's position in relation with Greenwich), and the number of the positions of the Sun's path. To establish the position of the panel in a time of day value is determined by the following calculations: the angle of the day, a correction factor of Earth's orbit, the solar declination angle, the equation of time in minutes, eastern time using latitude angle, the number of hours the Sun shines using angle eastern time, time the Sun sets, vectors containing the coordinates of the positions of the Sun (in this case 10 positions) during the day and azimuth angle. A Photovoltaic (PV) tracker system is one of those methods that are able to increase PV power generation. Theoretically, a PV tracker system with two axes can increase the overall solar energy capture by about $45 \%$, compared to a fixed PV module tilted at an angle equal to the local latitude. For a one-axis tracking system, the increase is approximately $32 \%$.
\end{abstract}

(C) 2017 The Author(s). Published by TAF Publishing.

\section{INTRODUCTION}

Renewable Energy Sources (RES) are getting more and more widespread, mainly due to the fact that they generate energy by keeping the environment clean. Their rapid evolution of RES during the last two decades materialised in a lot of RES power systems all over the world. A disadvantage of these power plants is the high cost of the installation. In this respect, the approaches trying to optimise the design of these ones are welcomed.

However, such an effort requires detailed knowledge, e.g. the meteorological data of the site where the system will be installed and operational results from similar systems, if available [1] and [2]. A PV tracker system is one of those methods that are able to increase the PV power generation. Theoretically, a PV tracker system with two axes can increase the overall solar energy capture by about $45 \%$, compared to a fixed PV module tilted at an angle equal to the local latitude. For one-axis tracking system, the increase is approximately $32 \%$ [3].

In this paper, we design and implement a two-axis tracking system of PV systems that follows the Maximum Power Point (MPP) using a programmable circuit XILINX type Complex Programmable Logic Device-CPLD and Xilinx ISE software. Thus, PV module will reach its MPP in relation to date and time of the day. The test bed relies on an algorithm integrated in the XILINX that has as inputs: date, location's latitude and longitude, the standard longitude (re-

\footnotetext{
${ }^{*}$ Corresponding author: Florin Dragomir

${ }^{\dagger}$ Email: drg_florin@yahoo.com
} 
lated to the location's position in relation with Greenwich), and the number the positions of the Sun's path.

Sun path refers to the apparent significant seasonaland-hourly positional changes of the sun (and length of daylight) as the Earth rotates, and orbits around the sun. The relative position of the sun is a major factor in the performance of solar energy systems. Accurate location-specific knowledge of sun path and climatic conditions is essential for economic decisions about solar collector area, orientation, landscaping, summer shading, and the cost-effective use of solar trackers. To gather solar energy effectively, a PV panel should be within about twenty degrees either side of perpendicular to the sun.

To establish the position of the panel in a time of day value is determined by the following calculations: the angle of the day, correction factor of Earth's orbit, the solar declination angle, the equation of time in minutes, eastern time using latitude angle, the number of hours the Sun shines using angle eastern time, time the Sun sets, vectors containing the coordinates of the positions of the Sun (in this case 10 positions) during the day and azimuth angle.

\section{SYSTEM ARHITECTURE}

Positioning on directions east-west and north-south of a solar panel (that is on a trajectory sun tracking device) is reduced to ordering automatic motor on the two axes of orientation system (Figure 1).

Position of the sun was calculated using formulas to approximate azimuth and elevation angles of the sun.

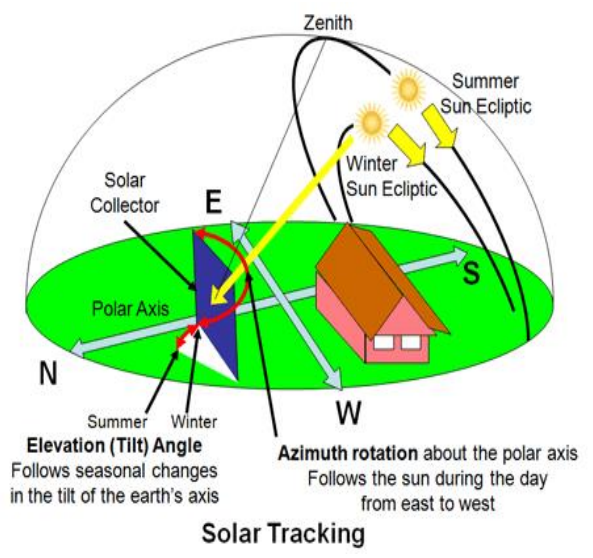

Fig. 1. Elevation angle and azimuth rotation [4]

To design and develop a prototype positioning for photovoltaic solar panels,performance conditions are useful table axis transducers to be $25 \mathrm{~kg}$, angular displace- ment speed up to $2 \mathrm{rev} / \mathrm{min}$ and limit switch heads with hardware method. Mechanical assembly for the vertical axis of rotation of the solar panel complies with kinematics scheme in Figure 2. In order to choose the electric motor you must know the operating conditions (work schedule, temperature and humidity environment), the required power and the rotation speed of the motor shaft. The motor-gearbox chosen to drive the axis is GP232004, manufactured by Baldor enterprise. The engine consumption must be low, and its torque must be multiplied by the mechanical transmissions [5].

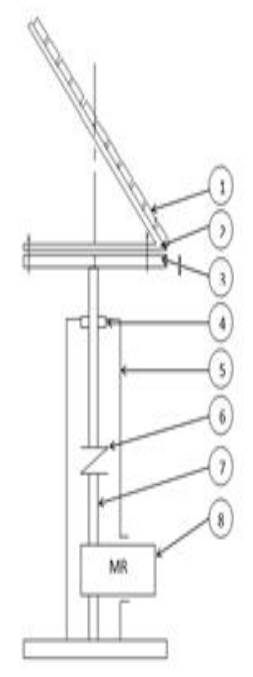

\author{
Assembly components are described below: \\ 1. Photovoltaic \\ 2. Support photovoltaic \\ 3. Turntable \\ 4. Roller bearing (thrust bearing) \\ 5. Housing / frame \\ 6. Flexible coupling \\ 7. Tree \\ 8. Motor-gearbox group
}

Fig. 2 . Cinematic scheme

The formula for calculating the approximate azimuth angle is shown in equation (1), where: $\Phi_{S}$ - azimuth angle; $\theta_{S}$ - elevation angle; $\Phi$ - declination of the sun; $\Phi$ local latitude.

$$
\cos \Phi_{S}=\frac{\sin \delta-\sin \sin _{S} \sin }{\cos \cos _{S}}
$$

Elevation angle is negative when the sun drops below the horizon. This angle may be approximated by the equation (2), where: $\mathrm{h}$ - hour angle; $\Theta_{S}$ - elevation angle; $\delta$ - declination of the sun; $\Phi$ - local latitude. [6]

$$
\sin \Theta=\cosh \cos \delta \cos \Phi+\sin \Theta \sin \Phi
$$

The hour angle of a point is the angle between two planes: one containing the Earth's axis and the zenith (the meridian plane), and the other containing the Earth's axis and the given point (the hour circle passing through the point). The angle may be expressed as negative east of the meridian plane and positive west of the meridian plane, 
or as positive westward from $0^{\circ}$ to $360^{\circ}$. The angle may be measured in degrees or in time, with $24 \mathrm{~h}=360^{\circ}$ exactly. For example for 10:30 AM, hour angle is -22.5 degrees (according to equation (3)).

$$
h=(\text { Time }-12) * 15+\text { minutes } * 0.25
$$

The declination of the sun is the angle that the sun's rays make with equatorial plane. This angle is shown in equation (4), where: $\mathrm{N}$ represents number of the day from current year and is calculated according to the equation (5).

$$
\delta 23.44 \sin
$$

$\mathrm{N}=$ int $(275 *$ month/9)-2*int $(($ month +9$) / 12)+$ day-30 (5)

The software was made in XILINX ISE and implements the algorithm for calculating the two angles of the sun showing two paintings:

- one for location data and is made up of several fields, for the following data: latitude, data from calendar (day / month / year) and local time in $24 \mathrm{~h}$ format (hour GMT +2 (hour / minute);

- and the second is for the results, and gives the calculated values of the declination angle, elevation, azimuth, azimuth east, sunrise in minutes and number of days of the year.

With this algorithm we can calculate the position of the sun for every day of the year with a good precision, so the voltage debited to photovoltaic panel is high. The mechanical implementation of solar tracker, which can move on east-west direction up to 270 garde was able to simulate the longest day of the year which was on June 21 (the summer solstice).

\section{A. XILINX ISE}

Software XILINX ISE (Integrated Software Environment) is the ideal solution for CPLD and FPGA design offering HDL synthesis and simulation, implementation, device fitting, and JTAG programming. To achieve an application can use schematic description or hardware description languages (VHDL or Verilog).

With this program can be achieved a synthesis application, which means behavioral model transformation made in a hardware description language (VHDL or Verilog) in a circuit structure.

\section{B. Complex Programmable Logic Devices Board}

A complex Programmable Logic Device (CPLD) is a programmable logic device with complexity between that of PALs and FPGAs, and architectural features of both [7] and [10]. The main building block of the CPLD is a macrocell, which contains logic implementing disjunctive normal form expressions and more specialized logic operations [6], [8], [9] and [11]. CPLDs have used analog sense amplifiers to boost the performance of their architectures. This performance boost came at the cost of very high current requirements. CoolRunner-II CPLDs, created by Xilinx, use an innovative all-digital core to achieve the same levels of performance at ultra-low power requirements. This allows designers to use the same CPLD architecture for both high-performance and low-power designs. CoolRunnerII CPLDs are the latest CPLD product offering from Xilinx. CoolRunner-II CPLDs combine high performance with low power operation. Standby current on CoolRunner-II CPLD devices is less than $100 \mu \mathrm{A}$.

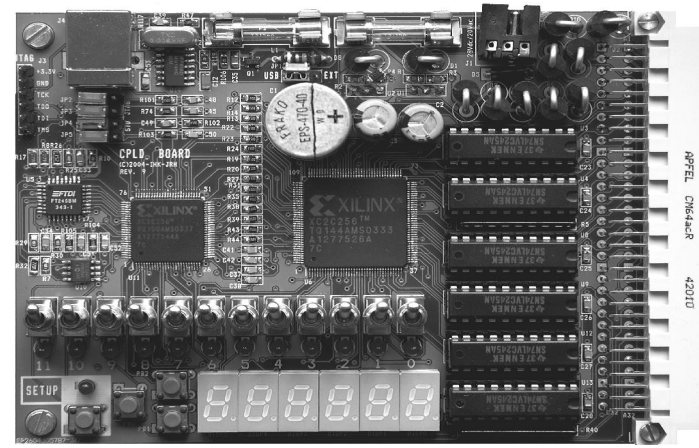

Fig. 3 . CPLD board

\section{APPLICATION DESCRIPTION}

Of all the elements that are available on board CPLD (Xilinx CoolRunner-II CPLD with 256 macrocells XC2C256-TQ144-7), in application presented are accessed as follows: Of all the elements that are available on board CPLD (Xilinx CoolRunner-II CPLD with 256 macrocells XC2C256-TQ144-7), in application presented are accessed as follows:

- 12 switches;

- 6 displays 7-segment (they will be displayed on the desired values);

- 1 button (reset), if we want the panel to return to its original position;

- clock signal which is variable ("ClkSlow") and the value is set at $1000 \mathrm{~Hz}$ in accordance with a setting in the configuration menu, then this value is used twice, is divided by two separator watches: one to obtain a correct display (visualization devices all appear to be lit at the same time) 
and one to obtain a delay when the panel moves from one position to another.

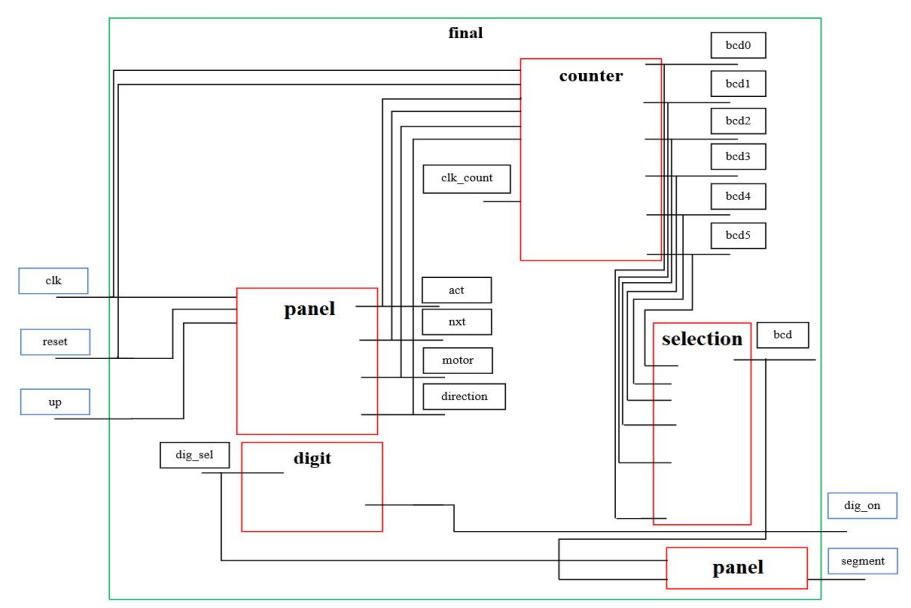

Fig. 4 . The main modules of the application

The panel will have 10 positions and may submit the following states:

- waiting in a position for changing the input, i.e. waiting for activation of the sensor corresponding with the motor in position stop; the panel in mod initial is in position 1 , that position being standard position;

- the photovoltaic panel can move from East to West, given command with help of the ten switches, or from West to East, that move will be achieved without an intervention when the panel reached its final position;

- the 10 buttons on the CPLD board, will be used for "up".
- the 6 displays on the CPLD board, will be used:

as 2 for position number (0-10);

a 3 for panel direction (ra - moving from East to West, ar-moving from West to East, PS - when it is in a certain position pending);

\& 1 for motor status (P-on, $\mathrm{O}$-off).

In Figure 5 are shown some states of the panel, and in Figure 6 are shown a few examples of transition states and change of the values of the variables used in the program.
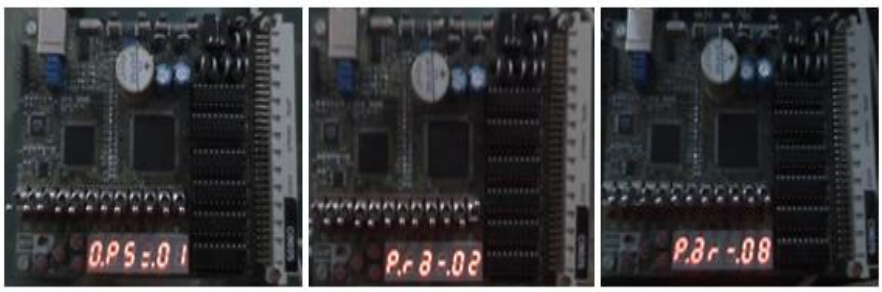

Fig. 5 . Representing the first state, the movement to the second position and the state of recovery of the panel

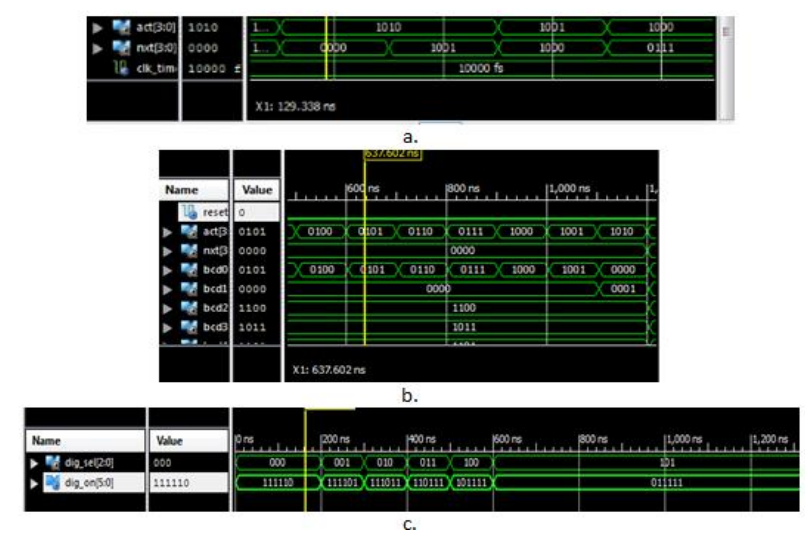

Fig. 6 . a. Transition states; b. Change of values bcd; c. Change the variable dig on 

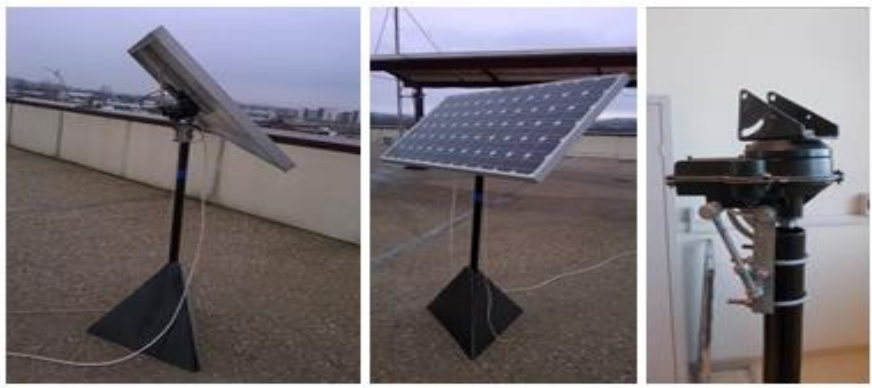

Fig. 7 . Power obtained by acquisition from photovoltaic panel

\section{EXPERIMENTAL RESULTS}

In Figure 7 is shown experimental application of "PV solar tracker" what describes the motion tracking of the Sun trajectory.
In the Figure 8 we have represented the power (P) debited of photovoltaic panel. Period of monitoring is in between the hours 07:05 and 17:35 on 17 February 2016.

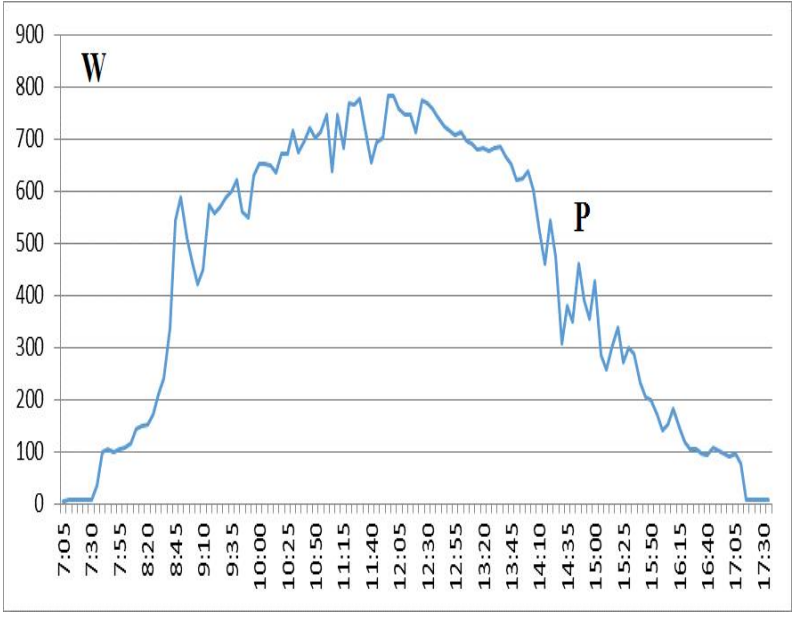

Fig. 8 . Power obtained by acquisition from photovoltaic panel

\section{CONCLUSION AND RECOMMENDATIONS}

The article solved an innovative problem related to solar energy capture systems and conversion into electricity. Permanent positioning of the solar panel after MPP point increases overall system efficiency. A photovoltaic tracker system is one of those methods that are able to increase the PV power generation.

The objective of the program made in Xilinx IDE was to describe the motion of a photovoltaic panel tracking the Sun trajectory. Thus, there was obtained a prototype of an algorithm for two-axis tracking photovoltaic panel that follows the MPP using a programmable circuit board (CPLD board) and Xilinx ISE software. Thus, photovoltaic panel will reach its MPP in relation to date and time of the day.
In a further development it is necessary to consider some aspect, such as the possibility of erroneous information from the sensors, due to adverse weather conditions.

The system will have an impact on the educational and research environment, being installed in the Multidisciplinary Science and Technology Research Institute of Valahia University of Targoviste and serving as teaching material for students of the Faculty of Electrical Engineering - Valahia University of Targoviste.

\section{ACKNOWLEDGEMENTS}

This work was supported by a grant of the Romanian National Authority for Scientific Research, CNDI- UEFISCDI, project code PN-II-PT-PCCA-2011-3.2-1616. 


\section{REFERENCES}

[1] World Energy Council, “World energy resources, 2013 Survey,” World Energy Council , London, UK, 2013.

[2] European Commission, "Communication from the Commission to the European Parliament, the Council, the Euro-

Economic and Social Committee and the Committee of the Regions, Renewable Energy: A major player in the European energy market," Brussels, Belgium, 2015.

[3] C. Y. Lee P. C. Chou, C. M. Chiang and C. F. Lin, "Sun tracking systems: A review," Sensors, vol. 9, no. 5, pp. 3875-3890, 2009. DOI: $10.3390 / \mathrm{s} 90503875$

[4] Woodbank Communications, "Battery and energy technologies, solar power (technology and economics)," [Online]. Available: goo.gl/JqMqmH

[5] A. Oprea and F. Dragomir, "Positioning a PV panel on a rotary axis after maximum light," Scientific Bulletin of Electrical Engineering Faculty, vol. 1, no. 21, pp. 14-18, 2013.

[6] F. Dragomir and S. Iliescu, Modelarea si Simularea Retelelor de Joasa Tensiune cu Producere Distribuita din Surse Regenabile de eEergie. Bucuresti, Romania: MatrixRom, 2013.

[7] Xilinx Inc., "CPLD. What is a CPLD?," [Online]. Available: goo.gl/Fxlk4K

[8] M. M. Hasan and O. Altun, "Two enhanced differential evaluation algorithms for expensive optimization," Journal of Applied and Physical Sciences, vol. 2, no. 2. pp. 48-53, 2016. DOI: 10.20469/ijtes.40002-4

[9] H. S. Alam, T. Haiyunnisa and Bahrudin, "Comparative analysis of P, Pi, and PID controller optimized by genetic algo-

on controlling drip irrigation system," International Journal of Technology and Engineering Studies, vol. 1, no. 4, 117-122, 2015. DOI: $10.20474 /$ japs-2.2.3

[10] P. Truttim and P. Sohsalam, "Comparison of electrocoagulation using iron and aluminium electrodes for biogas propp. tion wastewater treatment," Journal of Advances in Technology and Engineering Research, vol. 2, no. 2, pp. 35-40, 2016.

DOI: 10.20474 /jater-2.2.2

[11] B. S. K. Bhandary, K. P. Sharmila, N. S. Kumari, V. S. Bhat and G. Sanjeev, “Radioprotective potential of punica granaextract and synthetic ellagic acid: A biochemical and hematological study in mice," International Journal of Applied tum Physical Sciences, Vol. 1, no. 3, pp. 51-54, 2015. DOI: 10.20469/IJAPS.50001-3

— This article does not have any appendix. — 